\title{
Investigating the sensitivity of hurricane intensity and trajectory to sea surface temperatures using the regional model WRF
}

\author{
CeVahir KILIC ${ }^{1 *}$ and Christoph C. RAIBle ${ }^{1,2}$ \\ ${ }^{1}$ Climate and Environmental Physics, Physics Institute, University of Bern, Switzerland \\ ${ }^{2}$ Oeschger Centre for Climate Change Research, University of Bern, Switzerland
}

(Manuscript received February 1, 2013; in revised form September 11, 2013; accepted September 11, 2013)

\begin{abstract}
The influence of sea surface temperature (SST) anomalies on the hurricane characteristics are investigated in a set of sensitivity experiments employing the Weather Research and Forecasting (WRF) model. The idealised experiments are performed for the case of Hurricane Katrina in 2005. The first set of sensitivity experiments with basin-wide changes of the SST magnitude shows that the intensity goes along with changes in the SST, i.e., an increase in SST leads to an intensification of Katrina. Additionally, the trajectory is shifted to the west (east), with increasing (decreasing) SSTs. The main reason is a strengthening of the background flow. The second set of experiments investigates the influence of Loop Current eddies idealised by localised SST anomalies. The intensity of Hurricane Katrina is enhanced with increasing SSTs close to the core of a tropical cyclone. Negative nearby SST anomalies reduce the intensity. The trajectory only changes if positive SST anomalies are located west or north of the hurricane centre. In this case the hurricane is attracted by the SST anomaly which causes an additional moisture source and increased vertical winds.
\end{abstract}

Keywords: tropical cyclone, sea surface temperature, Loop Current eddies, Hurricane Katrina, WRF.

\section{Introduction}

It is well known that the sea surface temperature (SST) has an influence on the development and intensification of tropical cyclones (SHAPIRO and GOLDENBERG, 1998; TRENBERTH and SHEA, 2006; CiONE and UHLHORN, 2003; EMANUEL et al., 2004). This influence has become even more important during the past decades, as tropical cyclones show an intensification, which goes along with an increase in SSTs (EMANUEL, 2005). These empirical findings are confirmed by WEBSTER et al. (2005), SRIVER and HUBER (2006) and VECCHI and SODEN (2007). Among other processes, wind shear plays an important role in the intensification of a tropical cyclone (EMANUEL et al., 2004; GARNER et al., 2009). Clearly, the interplay of these different processes is complex and a focal point of the research concentrates on potential changes under future climate change. Most climate projections show that the number of tropical cyclones decreases in the warmer climate, whereas their intensity tends to increase (BENGTSSON et al., 1996; SUGI et al., 2002; EMANUEL et al., 2008; KNUTSON et al., 2010; RAIBLE et al., 2012).

\footnotetext{
${ }^{*}$ Corresponding author: Cevahir Kilic, Climate and Environmental Physics, Physics Institute, University of Bern, Sidlerstr. 5, 3012 Bern, Switzerland, e-mail: kilic@climate.unibe.ch
}

Recent studies with regional climate models (RCMs) have shown that SSTs have an effect on the intensity and the core speed of tropical cyclones (TCs). Using a case study approach, MANDAL et al. (2007) showed in RCM simulations that the enhancement of the storm intensity by positive SST gradients depends on the SST resolution, i.e., the storm intensity is enhanced with a higher resolution. They also found that the SST field and resolution has a notable impact on the TC trajectory. The TC tends to move towards higher SSTs. Additionally, the peak intensity of TC is reached over the highest SST. However, an explanation of how the SSTs influence the movement of the storm is not given in MANDAL et al. (2007). YUN et al. (2012) isolated the effects of SST magnitude and horizontal SST gradients in another case study using WRF (Weather Research and Forecasting) model in version 3.2 integrated over $72 \mathrm{~h}$ with a spatial resolution of $15 \mathrm{~km}$. They found that the movement of the TC, induced by the different SSTs in the east-west direction, is larger than in north-south direction. Further, the wavenumber-1 (WN1) potential vorticity tendency (PVT; WU and WANG (2000); CHAN et al. (2002)) is adopted to investigate the dynamical causes of the TC motion. The major finding is that the TC trajectory is shifted towards the area of maximum PVT. However, other processes such as the background wind field and the air-sea interaction needs to be considered to understand changes of the trajectory. 
Besides, SSTs in the Gulf of Mexico are affected by warm Caribbean water, the so-called Loop Currents, which provide a deep layer of very warm water (CHANG and OEY, 2013; ALVERA-AZCÁRATE et al., 2009). It is possible that an anticyclonic eddy of warm water separates from the main current and drifts to the west. These Loop Current eddies are able to serve as a huge energy source for TCs, which cross the Gulf of Mexico (HoNG et al., 2000).

The present study aims at systematically investigating the impact of basin-wide SST changes on tropical cyclones as well as the impact of Loop Current eddies, which are represented in an idealised way by localised SST anomalies. Therefore, we employ a regional numerical model and perform a series of sensitivity experiments in the case of Hurricane Katrina (August 2005). The present study further aims to provide insights into SST-TC interaction and is not focused on an accurate simulation of Hurricane Katrina. It should be understood as an idealised study. The analysis of the sensitivity experiments focuses on changes in intensity and trajectories with respect to a reference simulation.

The outline of this study is as follows: In Section 2 the model setup and the design of sensitivity experiments are briefly introduced. Then, the reference simulation is discussed and the results of the basin-wide and localised SSTs anomalies are presented (Section 3). Finally, the results are discussed and the main conclusions are given in Section 4.

\section{Model and experimental design}

In this study, we use the WRF model in its version 3.2.1 (SKAMAROCK et al., 2008) to generate a set of sensitivity experiments for Hurricane Katrina in 2005. The model is based on the fully compressible and non-hydrostatic Euler equations, which are solved on a two-way interactive, and quadruple-/quintuple-nested grid, where the inner nests move automatically to centre the hurricane.

Further, the microphysics scheme of HALL et al. (2005) is used and the Kain-Fritsch scheme is enabled for cumulus parameterisation (KAIN, 2004). Additionally, we employ a scheme based on Monin-Obukhov with Carlsong-Boland viscous sub-layer and standard similarity functions from look-up tables for the surface layer and the 5-layer thermal diffusion scheme. A non-local-K module with explicit entrainment layer and parabolic $K$ profile in unstable mixed layer is adopted to consider the planetary boundary layer physics. Longwave and shortwave radiation are taken into account by the rrtmg and Dudhia scheme. The corresponding physic modules include cloud effects and surface fluxes. The longwave radiation module is the new version of the Rapid Radiative Transfer Model which is available since version 3.1.
The Dudhia scheme is responsible for the shortwave radiation. The surface roughness is provided by a lookup table, where the value for water is $10^{-4} \mathrm{~m}$. At high wind speeds the surface roughness follows the Donelan parameterisation, which has the advantage that the drag coefficient reaches its maximum at about hurricane wind speeds, while the drag coefficient according to Charnock parameterisation increases with no limit.

To initialise the WRF model, we use the National Center for Environmental Prediction and the National Center for Atmospheric Research (NCEP/NCAR) reanalysis data set (KALNAY et al., 1996; KISTLER et al., 2001). The reanalysis system utilises the operational NCEP/ NCAR global spectral model with a horizontal resolution of T62 (triangular truncation of 62 waves corresponding to a regular longitude-latitude grid of approximately $2^{\circ} \times 2^{\circ}$ equivalent to about $210 \mathrm{~km}$ ) and 28 vertical levels (sigma levels). The SST fields are also obtained from the NCEP/NCAR reanalysis data set. We use the data provided on a $2.5^{\circ} \times 2.5^{\circ}$ regular grid with 18 vertical levels. Note that the major impact of TC-SST interaction is a cooling of SST by TC. This interaction can lead to a decrease in SST by approximately $5 \mathrm{~K}$, as strong winds, caused by the TC, upwell cooler water from deeper layers to the warm surface. Additionally, latent and sensible heat fluxes from the ocean to the atmosphere underneath a TC core can lead to a cooling of the surface. However, these cooling processes are not present in the reanalysis data, due to the coarse resolution. Moreover, the SST field is fixed to the initialisation time during the entire simulation (Fig. 1a).

Different setups of the horizontal and vertical resolution and the number of nests are tested. We find major improvements of the inner core structure of a hurricane (e.g., eye and eye wall) from $12 \mathrm{~km}$ to $4 \mathrm{~km}$ in a quadruple-nested grid, whereas a further increase to $1.33 \mathrm{~km}$ using a quintuple-nested grid does not significantly improve the basic structure of the hurricane. Additionally, a doubling of the vertical levels (eta levels) from 36 to 72 (using the quadruple-nested grid up to $4 \mathrm{~km}$ ) shows no significant change in important hurricane characteristics, such as intensity or track. Thus, the reference simulation and the sensitivity experiments are carried out with a quadruple-nested grid, with the resolutions $108 / 36 / 12 / 4 \mathrm{~km}$ $(60 \times 60 / 121 \times 121 / 301 \times 301 / 400 \times 400$ grid points $)$ and on 36 full eta levels in the vertical $(1,0.993$, $0.983,0.97,0.954,0.934,0.909,0.88,0.839,0.798$, $0.757,0.716,0.644,0.578,0.517,0.461,0.411,0.364$ $0.322,0.283,0.248,0.216,0.188,0.162,0.138,0.117$ $0.098,0.081,0.066,0.053,0.041,0.03,0.021,0.013$, $0.006,0)$, where the boundary layer is at $1.0 \geq 0.9$, the lower layer at $0.9 \geq 0.55$, and the upper layer at $0.55 \geq 0.25$. The model is integrated over $72 \mathrm{~h}$ using the time steps $180 \mathrm{~s}, 60 \mathrm{~s}, 20 \mathrm{~s}$, and $6.67 \mathrm{~s}$ for the different nests. 
(a)

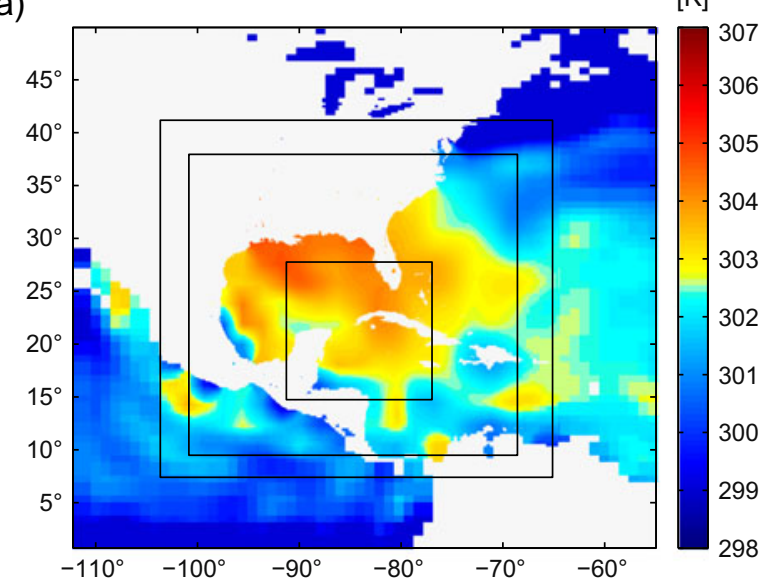

(b)

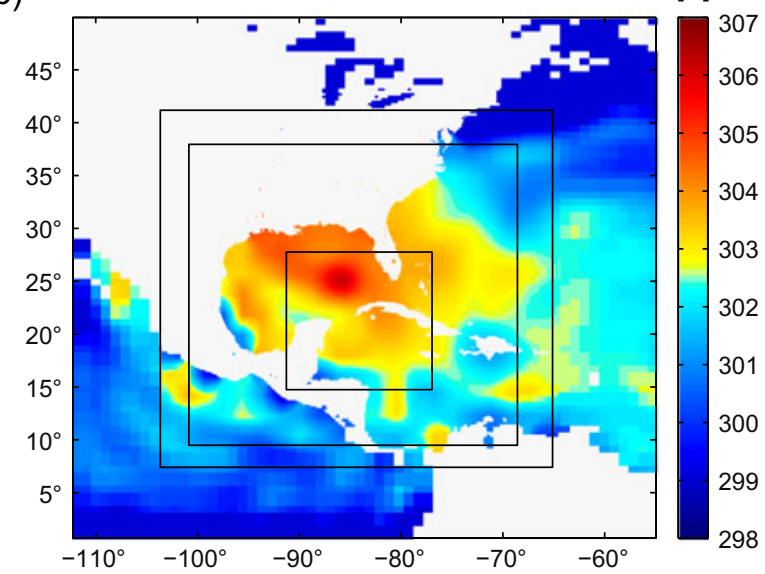

(c)

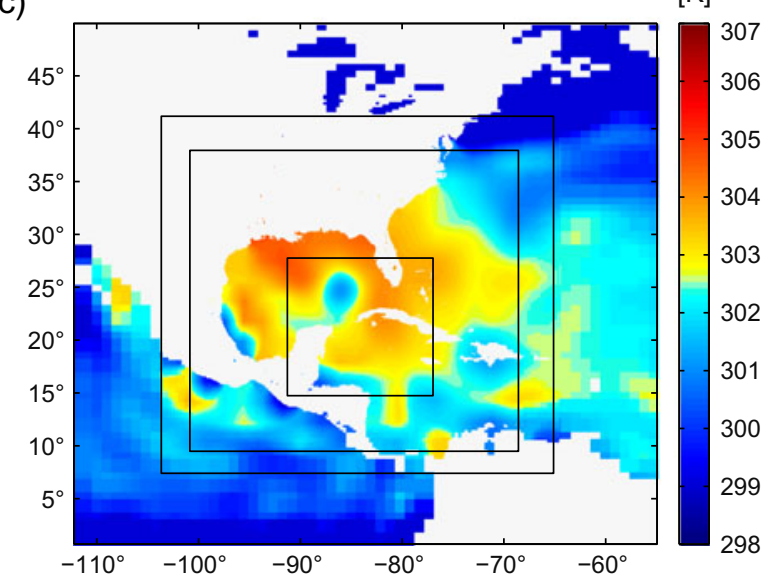

Figure 1: Sea surface temperature (a) of the reference simulation, (b) with a positive localised anomaly of $+2.5 \mathrm{~K}$, and (c) with a negative localised anomaly of $-2.5 \mathrm{~K}$ at $86^{\circ} \mathrm{W} 25^{\circ} \mathrm{N}$, respectively. The nest positions are at the point of initialisation.

The reanalysis is rather coarsely resolved and therefore a hurricane is strongly underestimated in its strength and extent and may even not be detectable (KLEPPEK et al., 2008; RAIBLE et al., 2012). Thus, there is a need to initialize a vortex in order to be able to simulate a hurricane. We favor a rather simple vortex initialisation, i.e., the bogus vortex initialisation (WANG et al., 2008;

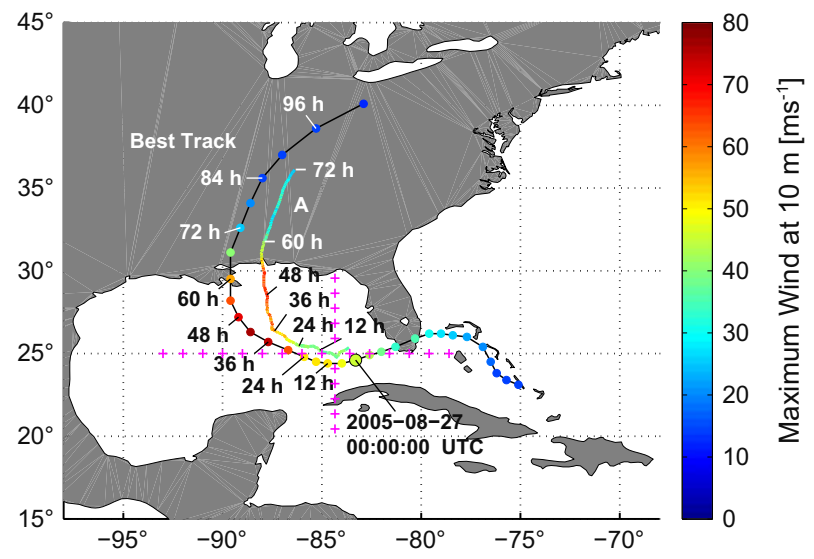

Figure 2: Trajectory and intensity (maximum wind at $10 \mathrm{~m}$ ) of Hurricane Katrina according to the best track data and to the reference simulation initialised on 27.08.2005 at 00:00:00 UTC. Best track provides a $6 \mathrm{~h}$ time resolution, which are indicated by circles, whereas the reference simulation provides a 15 min time resolution. The locations of the SST anomalies are denoted with + .

LOW-NAM and DAVIS, 2001, 2009; FREDRICK et al., 2009), which is sufficient to perform sensitivity studies. By using this bogussing initialisation scheme it is, however, not possible to simulate the observed trajectory and intensity. Hence, deviations in intensity and trajectory are expected due to this simple vortex initialisation scheme. The bogussing scheme is a two-step procedure: First, the existing vortex from the input data is removed, i.e., wind field, temperature, pressure, and moisture are adjusted to the environment. Second, a new wind field is inserted based on a simple Rankine vortex (LOW-NAM and DAVIS, 2009). This Rankine vortex uses coordinates of the vortex centre, radius of maximum wind, and maximum wind speed from the best track data which are best estimates of storm positions and intensities at 6-hourly intervals. The best track data are generated by reanalysing all available data such as satellite, surface, and ship observations (KNAPP et al., 2010). Additionally, the relative humidity is considered as well in the bogussing scheme: The humidity is set to $95 \%$ inside the radius of maximum wind. Between the radius of maximum wind and twice its value, the relative humidity decreases linearly to the mean humidity of the input data.

To assess the sensitivity of hurricane characteristics to SST changes, a reference experiment and two sets of sensitivity experiments are performed, which are based on the case Hurricane Katrina. Hurricane Katrina is selected as its trajectory is centred over the Gulf of Mexico. It formed on 23 August 2005 over the Bahamas, hit Florida on 25 August as a Category 1 storm. After that, Katrina strengthened in just 12 hours over the Gulf of Mexico to a Category 5 hurricane and hit the US-Gulf coast on 29 August as Category 3 hurricane (Fig. 2). The maximum sustained wind speed at $10-\mathrm{m}$ reached $78 \mathrm{~m} \mathrm{~s}^{-1}$ with a central pressure of $902 \mathrm{hPa}$ on 28 August 2005 . 
The magnitude of the radius of maximum wind extended to $20-100 \mathrm{~km}$.

The reference simulation is initialised on 27.08 .2005 at $00 \mathrm{UTC}$ at $84.34^{\circ} \mathrm{W} 24.712^{\circ} \mathrm{N}$. This date is selected, as Hurricane Katrina is just before its most intense state and will reach the coastline within 60 hours. The bogussing vortex is initialised with a maximum wind speed of $135 \mathrm{kn}$ and a radius of maximum wind of almost $28 \mathrm{~km}$.

In the first set of sensitivity experiments the entire SST field in the Atlantic basin is varied by adding uniformly $-2.5 \mathrm{~K}$ to $+2.5 \mathrm{~K}$ (using $0.5 \mathrm{~K}$ intervals). This results in 10 sensitivity experiments.

The second set of sensitivity experiments uses a localised SST anomaly, which is implemented at different positions south to north and east to west of the initial position of Hurricane Katrina. The SST anomalies are given by a two-dimensional Gaussian function

$$
f(x, y)=A e^{-\left(\frac{\left(x-x_{0}\right)^{2}}{2 \sigma_{x}^{2}}+\frac{\left(y-y_{0}\right)^{2}}{2 \sigma_{y}^{2}}\right)},
$$

where the coefficient $A$ is the amplitude, $x_{0}$ and $y_{0}$ are the coordinates of the centre, $\sigma_{x}$ and $\sigma_{y}$ are the standard deviations in $x$ and $y$ direction. Since we use latitudes and longitudes, the parameters $\sigma_{x}$ and $\sigma_{y}$ do not have the exact same values, but they are set in such a way that $\sigma_{x}$ and $\sigma_{y}$ correspond to $150 \mathrm{~km}$. The centre of the anomalies is set westwards, eastwards, southwards, and northwards with respect to the point of initialisation. The distance between each anomaly centre is approximately $110 \mathrm{~km}$. We place nine anomalies westwards, six eastwards, five southwards, and five northwards. Fig. 2 indicates, inter alia, the locations of the SST anomalies. Additionally, the amplitudes of the SST anomalies $A$ are varied using values of $-5 \mathrm{~K},-2.5 \mathrm{~K},+2.5 \mathrm{~K}$, and $+5 \mathrm{~K}$ as illustrated by two examples in Fig. 1b,c. Thus, we simulate a total of one hundred sensitivity experiments using localised SST anomalies.

\section{Results}

\subsection{The reference simulation}

The trajectory and intensity of the reference simulation are compared with the best track data of Hurricane Katrina (Fig. 2). One obvious discrepancy is that the initialisation point does not fully correspond to the best track data. The reason is that we initialize the hurricane where the vortex is located in the reanalysis data. This is necessary, because the vortex in the input data is not fully damped by the two-step bogussing scheme and a superposition of the remaining vortex and the bogus vortex could lead to numerical instabilities.

Comparing the reference simulation with the best track data we find that the intensification prior to the land fall is to some extent realistically simulated (Fig. 2). The subsequent weakening is also reflected in the reference

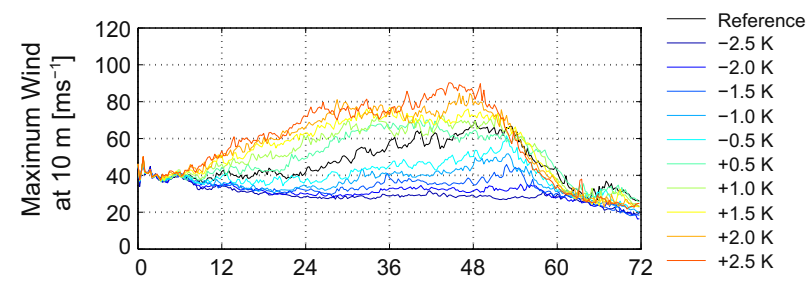

Figure 3: Time series of maximum wind at $10 \mathrm{~m}\left(\mathrm{~m} \mathrm{~s}^{-1}\right)$ of the basin-wide SST sensitivity experiments (colour) and the reference simulation (black). The SST of the entire ocean changes from $-2.5 \mathrm{~K}$ to $+2.5 \mathrm{~K}$, with respect to the reference simulation, using $0.5 \mathrm{~K}$ intervals.

simulation. Note that the first 4-5 hours are dominated by effects of the initialisation and should not be interpreted. The simulated track deviates to some extent from the observed one mainly due to the initialisation method. It is shifted eastwards, i.e., the simulated vortex moves earlier to the north than the observed track. Nevertheless, the basic structure of trajectory is similar to the observed one.

It is important for the sensitivity experiments that the trajectory and intensity are insensitive to small perturbations. A hint whether the reference simulation fulfills this requirement is given by the test simulations for the vertical and horizontal resolutions and the appropriate time step. All tests show that the trajectory is not affected by the horizontal or vertical resolution and only slightly affected by the time step. An increased time step enables the bogus vortex to adapt faster to the background flow and thus the trajectory is slightly shifted to the east. The intensity is insensitive to the time step, but, as expected, sensitive to the horizontal resolution; e.g., a 12-km resolution leads to a strong underestimation of the maximum intensity. However, resolutions higher than $4 \mathrm{~km}$ show no substantial changes. Thus, small perturbations do not lead to substantial changes of the trajectory and intensity.

Overall, the reference simulation shows that we are able to simulate the important characteristics of Hurricane Katrina with the rather simple bogussing initialisation. Deviations from the best track data are expected, as no data in the model domain are assimilated during the simulation. These deviations are systematic biases and have no implications in the sensitivity experiments.

\subsection{Sensitivity to basin-wide SST changes}

The sensitivity experiments with a basin-wide SST change give a first hint how a TC like Hurricane Katrina would react in a colder or warmer world than today. The results show that the intensity is strongly connected to the underlying SST: A higher SST than the one in the reference simulation causes an intensification, whereas reduced SSTs lead to a weakening of the vortex (Fig. 3). This is an expected result due to the thermodynamic changes. EMANUEL (1997) showed that the 


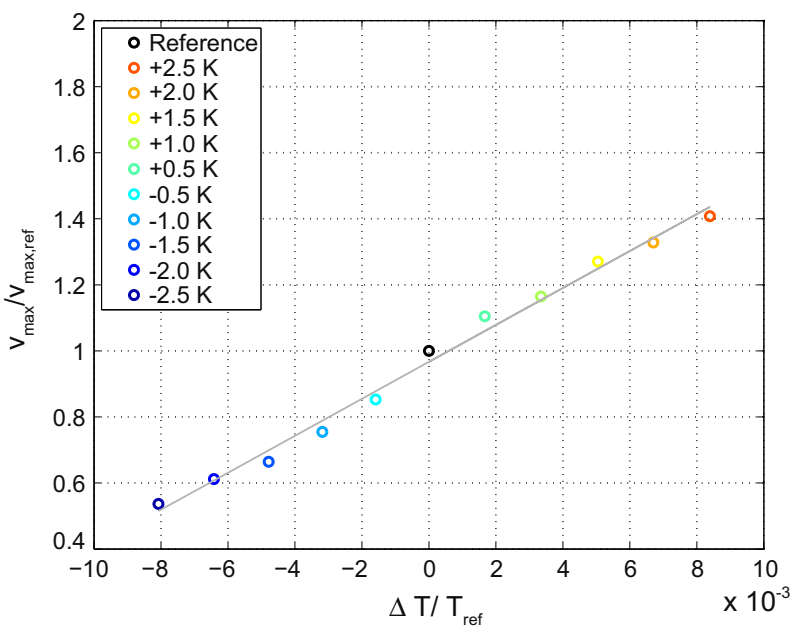

Figure 4: Relation between relative intensity $\left(V_{\max } / V_{\max , r e f}\right.$ using $10-\mathrm{m}$ wind) and the relative SST change $\left(\Delta T / T_{\text {ref }}\right)$ underneath the TC core. The ratios are averaged between $12 \mathrm{~h}$ and $48 \mathrm{~h}$ of the simulation time.

relation between SST and TC energy follows a Carnot cycle. The mechanical energy, which could be interpreted as intensity, roughly scales with the temperature difference of SST and outflow temperature at the top of the TC. Moreover, high SSTs facilitate evaporation, which is another important process delivering energy for a tropical cyclone. Our simulations confirm the findings of EMANUEL (1997) and show a linear increase in storm intensity from about $301 \mathrm{~K}$ up to $307 \mathrm{~K}$ (Fig. 4).

The trajectory of the vortex also reacts to mean SST changes (Fig. 5). A reduction of the SSTs with respect to the reference simulation induces an eastwards shift, except for simulations, where the SST are decreased by $-2.5 \mathrm{~K}$ and $-2 \mathrm{~K}$ (Fig. 5). In these $-2.5 \mathrm{~K}$ and $-2 \mathrm{~K}$ experiments, the vortex moves to New Orleans, but prior to landfall the vortex turns to the northeast. The reason for this change in the trajectory is the low intensity of about $30 \mathrm{~m} \mathrm{~s}^{-1}$ or less (10-m wind speed). For positive SSTs the trajectory shifts more to the west with respect to the reference simulation. One potential reason for the westwards shift induced by the positive SST forcing is an impact on the speed of the vortex. However, we find no correspondence between an increase in SSTs and the speed of the vortex. Another possibility is that the mean flow in the tropical Atlantic is affected by changing the underlying SST field and that this change causes a more westerly trajectory of tropical cyclones when uniformly increasing the SSTs. To investigate potential changes of the mean flow, the zonal wind fields are averaged up to $850 \mathrm{hPa}$. Comparing the fields of the sensitivity experiments with a uniform positive SST increase to the reference experiment shows that the ocean warming causes stronger easterly winds which move the vortex to the west, whereas a uniform decrease of SSTs damps the easterly winds; thus the vortex is shifted to the east relative to the reference experiments.

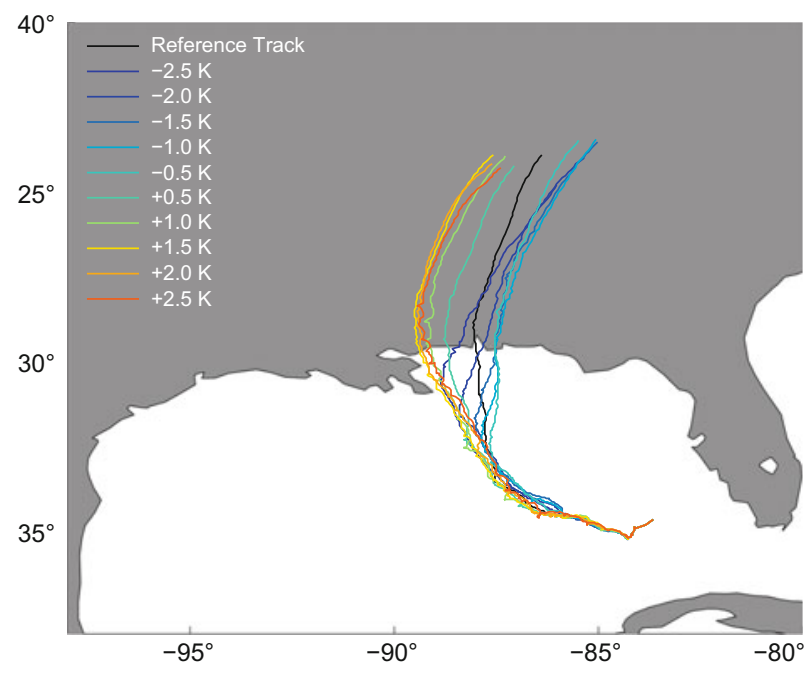

Figure 5: Hurricane trajectories of the basin-wide SST sensitivity experiments (colour) and the reference simulation (black). The SST of the entire ocean changes from $-2.5 \mathrm{~K}$ to $+2.5 \mathrm{~K}$, with respect to the reference simulation, using $0.5 \mathrm{~K}$ intervals.

To gain further insights in the dynamics, the potential vorticity (PV) and its tendency (PVT) are analysed (definitions in Appendix A). In terms of PV, a TC can be treated as a strong localised three-dimensional positive anomaly in the lower and middle troposphere with a complex PV pattern in the upper outflow layer (WU and WANG, 2000; SHAPIRO and FRANKLIN, 1995). Hence, the TC and the localised positive PV anomaly movement are equal and, so, the investigation of the PV anomaly movement is used to deepen our understanding of the contributions of the various physical processes. Similar to the findings of WU and WANG (2000), CHAN et al. (2002), and YUN et al. (2012), a positive PVT is located to the moving direction relative to the TC centre. Splitting the PVT in the horizontal advection, vertical advection, and diabatic heating terms (Appendix A), we find that mainly the horizontal advection term contributes to this PVT maximum, due to a steering by strong environmental flow (Fig. 6a). The impact of the diabatic heating is of minor importance (Fig. 6b), and hence, the TC motion is dominated by horizontal advection (YUN et al., 2012; CHAN et al., 2002). The amount of the horizontal advection (Fig. 6a-c) as well as the amount of the diabatic heating (Fig. 6d-e) rise with increasing SST due to the enhanced Carnot cycle.

\subsection{Sensitivity to localised SST changes}

Additionally to the basin-wide SST experiments, we investigate the response of TCs to localised SST variations in the second set of sensitivity experiments in order to explore the impact of Loop Current eddies in the Gulf of Mexico on TCs. This set is summarised in Fig. 7 by showing the relation between the position of the SST anomaly relative to the initialisation point of the reference simulation and the intensity as well as two landfall 


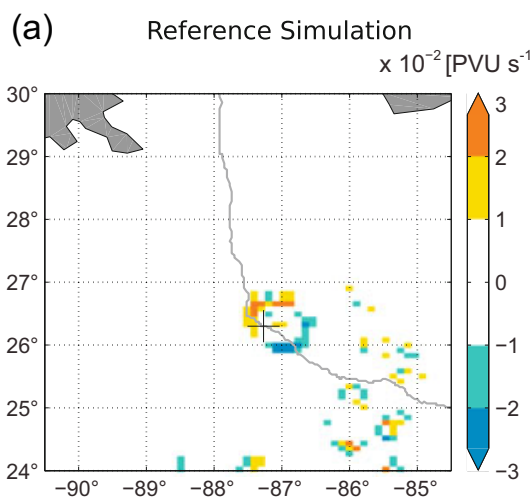

(b) $\quad+2.5 \mathrm{~K}$ Experiment

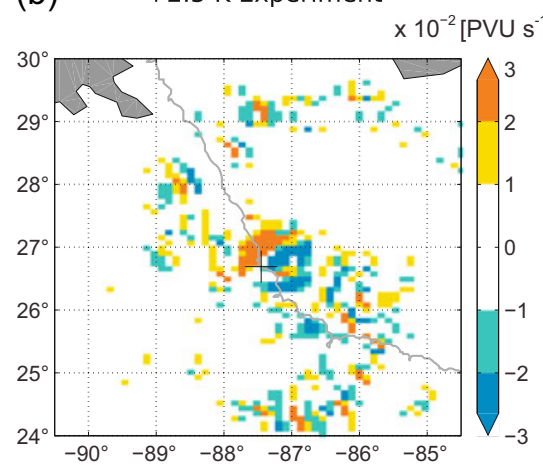

(c)

$-2.5 \mathrm{~K}$ Experiment

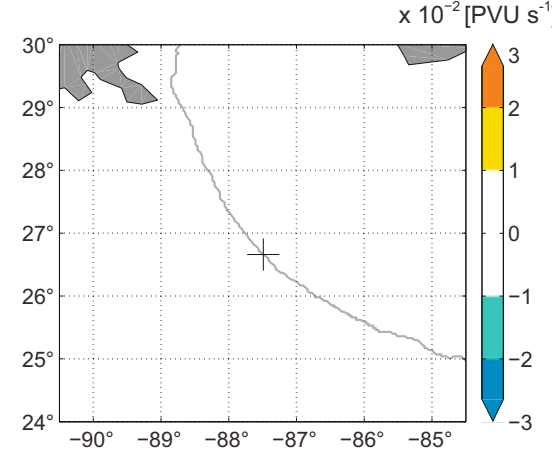

(d)

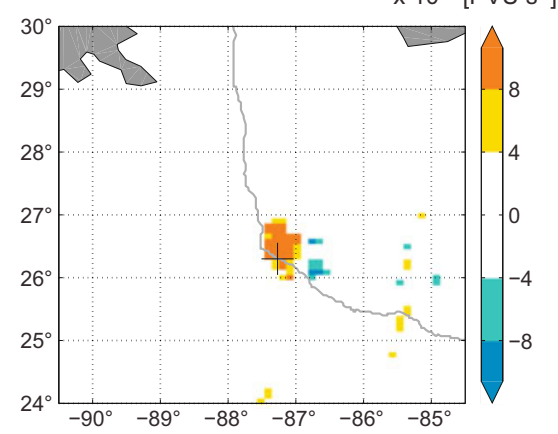

(e)

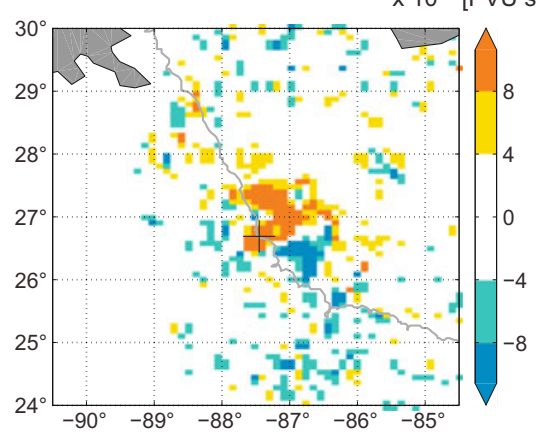

(f)

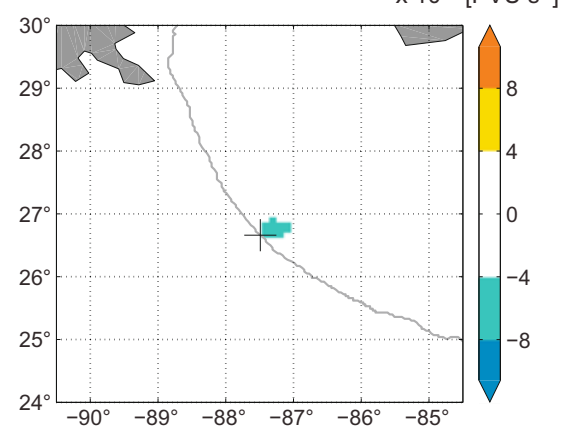

Figure 6: Horizontal advection (a,b,c) and diabatic heating (d,e,f) averaged over the lower layer (see Section 2 ) at $36 \mathrm{~h}$ after simulation starts, shown for the reference simulation, $+2.5 \mathrm{~K}$ experiment, and $-2.5 \mathrm{~K}$ experiment. Please note that the colour bars deviate by three orders of magnitude for a-c compared to d-f.

parameters (location in west east direction and timing). The intensity, measured by the $10-\mathrm{m}$ wind speed averaged between $12 \mathrm{~h}$ and $48 \mathrm{~h}$ after initialisation, strongly depends on the sign, the strength and the position of the SST anomaly and can be treated by an oneor two-dimensional Gaussian function (Fig. 7a,b), since the localised SST anomalies are Gaussian distributed and a linear relation between intensity and SST is present. Applying a positive (negative) SST anomaly within a range of $-5^{\circ}$ to $+3^{\circ}$ relative to the initialisation point in west-east direction and $-3^{\circ}$ to $+4^{\circ}$ in south-north direction leads to an intensification (weakening) of the hurricane. Additionally, we find that in contrast to a steady intensification for the positive SST anomalies the weakening is similar for -2.5 and $-5 \mathrm{~K} \mathrm{SST}$ anomalies being a hint for a nonlinear or threshold-type behavior.

Besides, the landfall of the hurricane is affected by localised SST anomalies (Fig. 7c-f). As the US coastline in the Gulf of Mexico is nearly east-west oriented, we only investigate, the position of landfall in this direction (Fig. 7c-d). For the negative SST anomalies we find a relatively weak response, i.e., a slight eastwards shift of the landfall, which is nearly independent from the position of the SST anomaly. The experiments with positive SST anomalies show a strong westwards shift of the landfall, in particular when the SST anomaly is located in the west and to some extent in the north. Again, this fits to the trajectory of the hurricane with its south-east to north-west orientation. Moreover, the timing of the landfall is affected by the localised SST anomalies showing that the hurricane makes landfall 5-6 hours earlier for the $+5 \mathrm{~K}$ SST anomaly experiments, which are located nearby the initialisation point of the reference simulation (Fig. 7e-f). This is clearly driven by the intensification and the acceleration of the hurricane. Note that the 1-2 hours earlier landfall of the negative SST anomaly experiments is due to slightly more western landfall and the associated coastline changes.

As for the basin-wide experiments, the relation between TC intensity and SST is investigated for all localised SST anomaly experiments (Fig. 8). Contrary to the basin-wide SST experiments, there is no obvious linear relation between intensity and temperature using all experiments. However, if only considering experiments with SST anomalies at the same location, a linear relation between temperature and TC intensity is present from $-2.5 \mathrm{~K}$ to $+5 \mathrm{~K}$. The gradient decreases with increasing distance between SST anomaly and initialisation point, and it also depends on the orientation of the SST anomaly with respect to the TC. The anomalies located west and north of the initialisation point have a stronger impact than the ones located south and east, as they lie in the moving direction of the TC. The experiments with a far distance SST anomaly (e.g. $>\Delta 6^{\circ}$ for west localised anomalies) have no significant influence on TC intensity, hence, the corresponding values just spread around the reference simulation. 
(a)

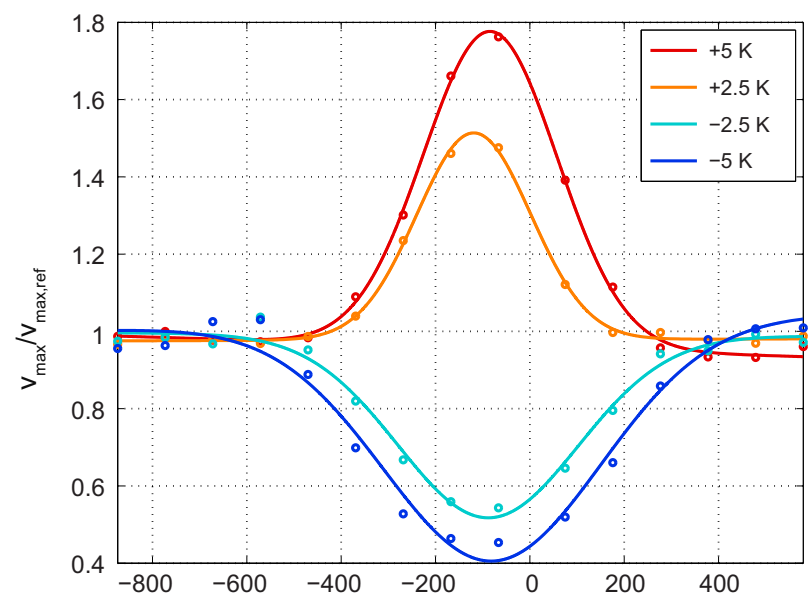

(c)

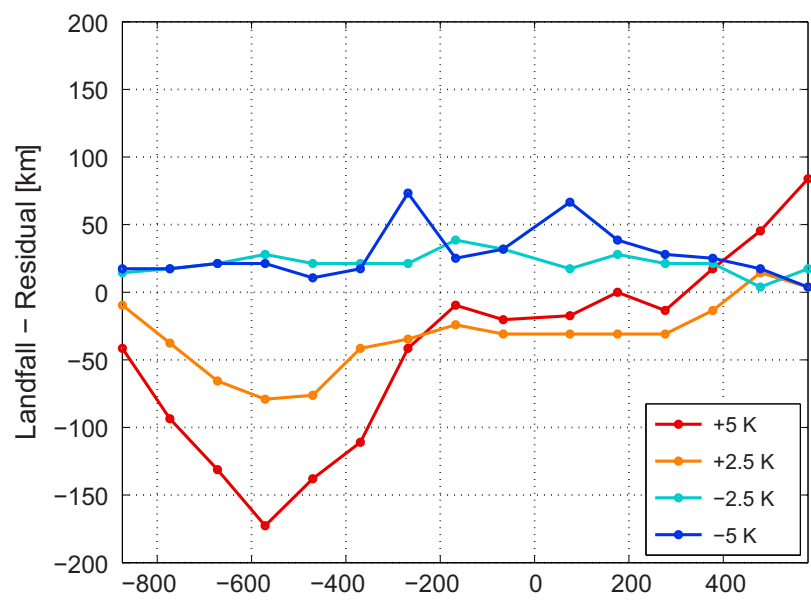

(e)

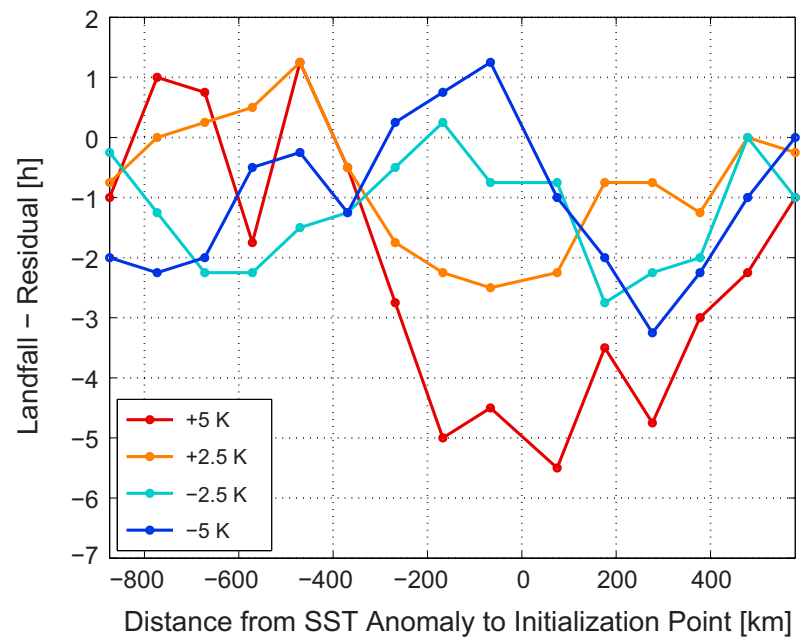

(b)

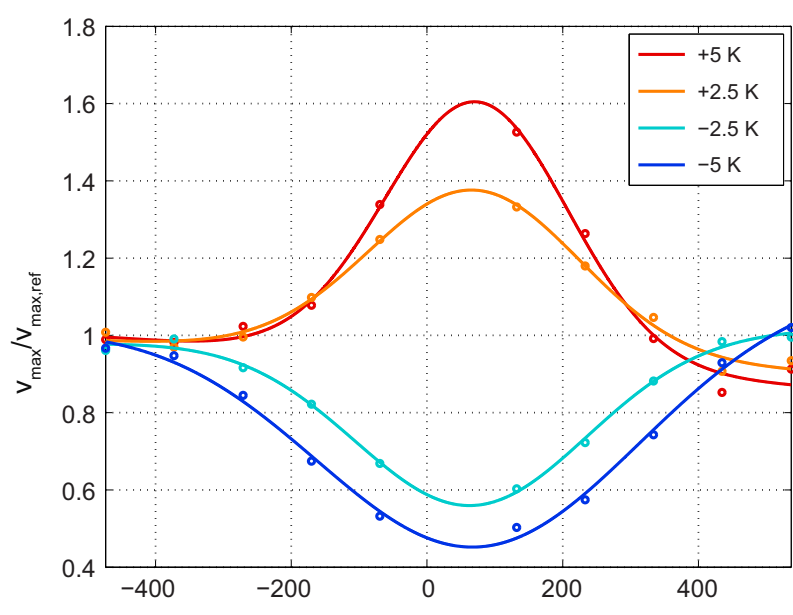

(d)

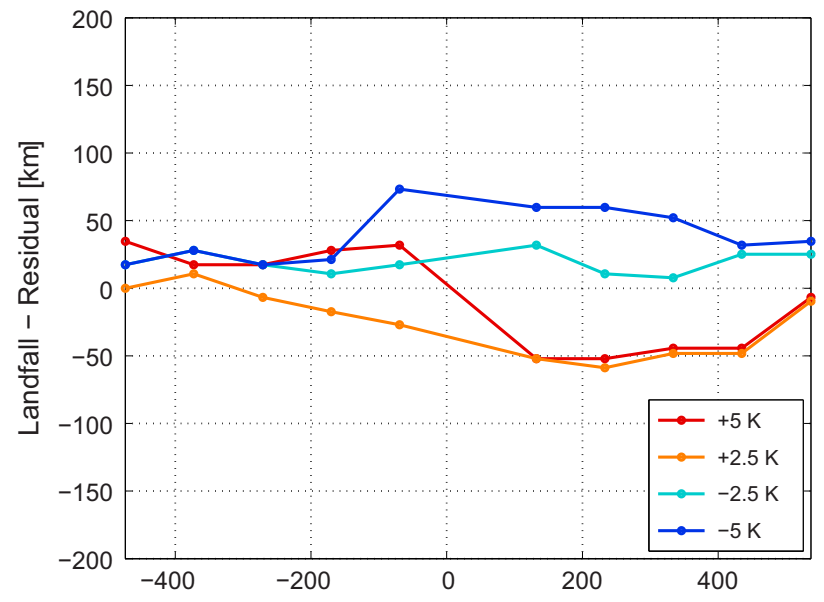

(f)

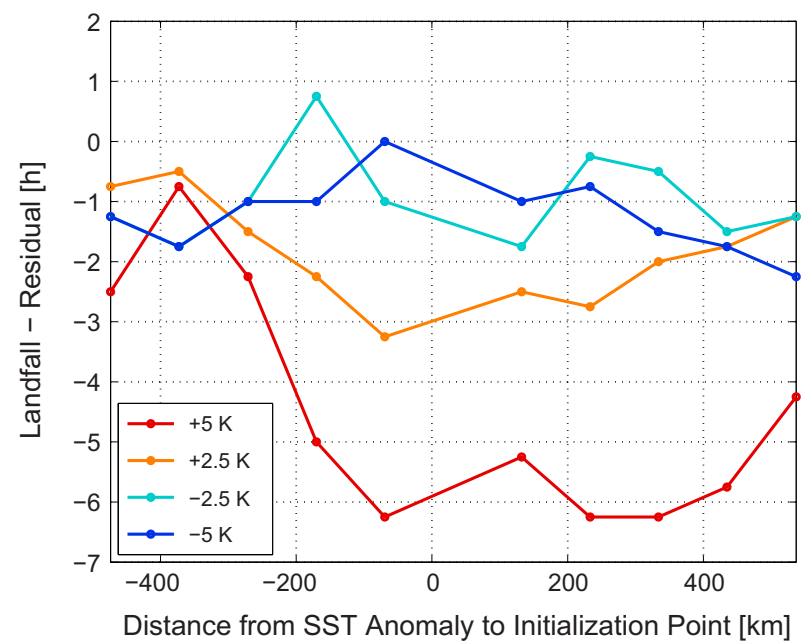

Figure 7: Relation between distance of the SST anomaly to the initialisation point and (a,b) ratio of intensity (10-m wind speed) averaged from $12 \mathrm{~h}$ to $48 \mathrm{~h}$ after initialisation supplemented with Gaussian fits, (c,d) landfall in west-east direction, and (e,f) the timing of the landfall. The distance is either in west-east direction (left column) or in south-north direction (right column). The residuals are given relative to the reference simulation. 


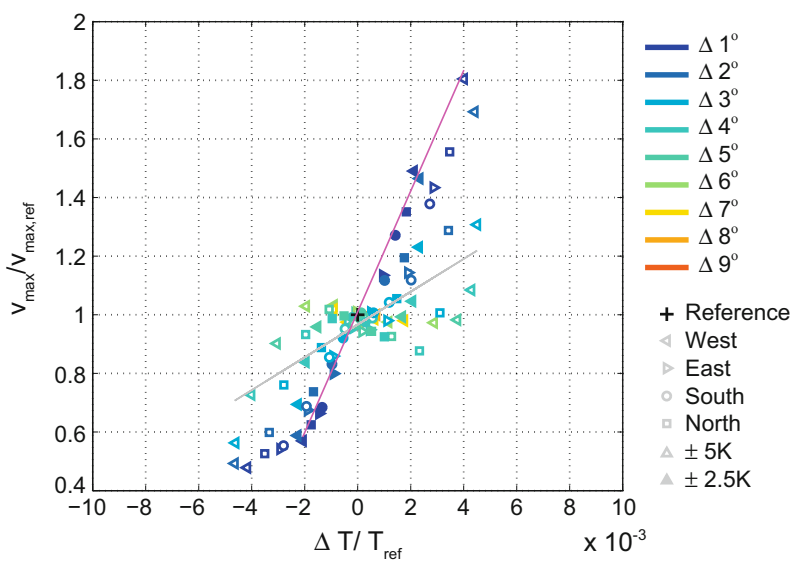

Figure 8: As Fig. 4, but for all localised SST anomaly experiments. The colour indicates the distance to the initialisation point, the shape of the symbols represents the direction, and the filled/unfilled symbols are used to distinguish the amount of SST anomaly amplitude. The values left to the reference value correspond to the experiments with a negative SST anomaly amplitude and the values right to the reference value to the experiments with a positive one. The linear relation of the $\Delta 1^{\circ}$ west experiments (magenta) is given: For comparison, the linear relation of the basin-wide SST experiments (grey) from Fig. 4 is shown as well.

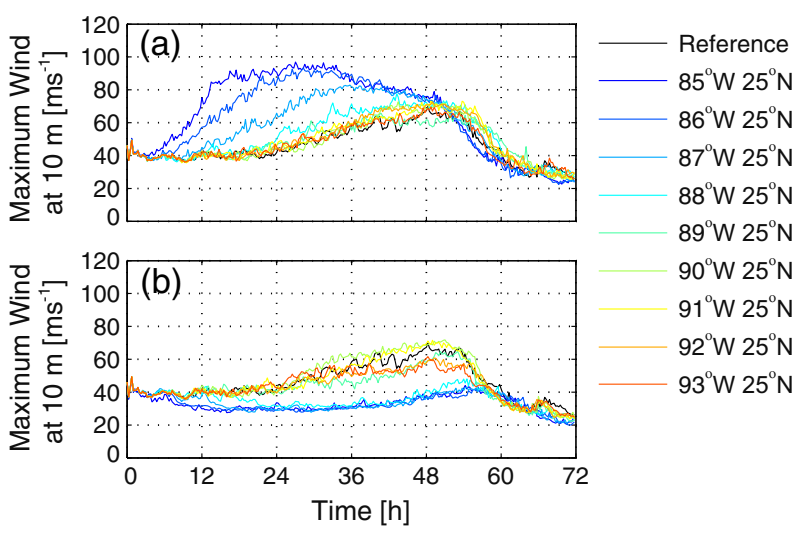

Figure 9: Time series of maximum wind at $10 \mathrm{~m}$ of the reference simulation and the westwards localised SST experiments using an amplitude of (a) $+5 \mathrm{~K}$, and (b) $-5 \mathrm{~K}$.

An interesting point is that the same average SST does not lead to the same intensity, e.g., the experiments located $\Delta 1^{\circ}$ and $\Delta 6^{\circ}$ west of the initialisation point show a similar $\Delta T /$ $T_{\text {ref }}$ however, strongly deviate in intensity. Hence, the location or timing, when the TC is affected by a localised SST anomaly, is crucial: The impact of a localised SST anomaly on the intensity is larger, if the TC crosses the anomaly centre and, if the anomaly is localised enough far from the coast to enable the time needed for TC development.

To gain further insight in the response of intensity and trajectory to localised SST anomalies we focus in the following on the experiments with $\pm 5 \mathrm{~K}$ SST anomalies, which are located westwards to initialisation point. In Fig. 9a,b the time series of the intensity is presented. Positive anomalies, which are located close to the initialisation (a)

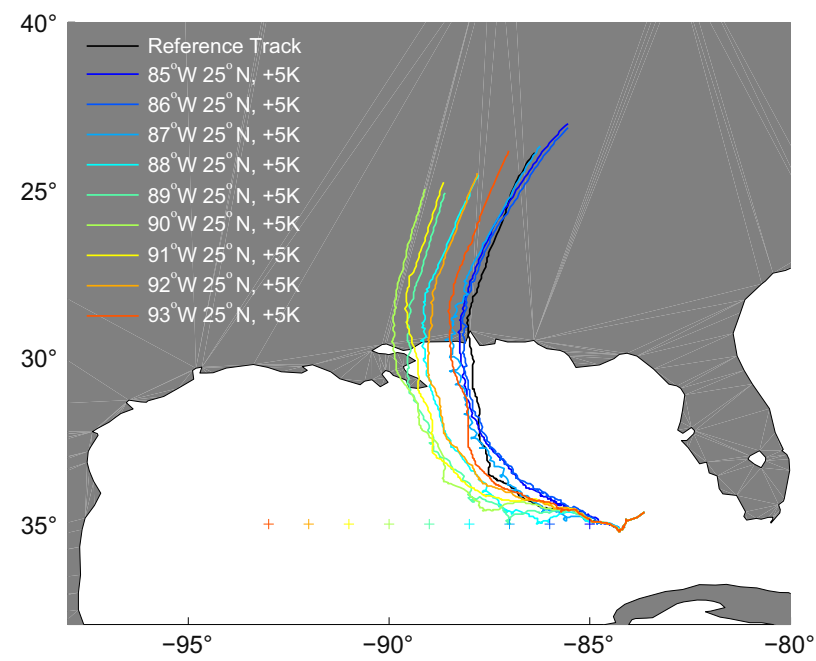

(b)

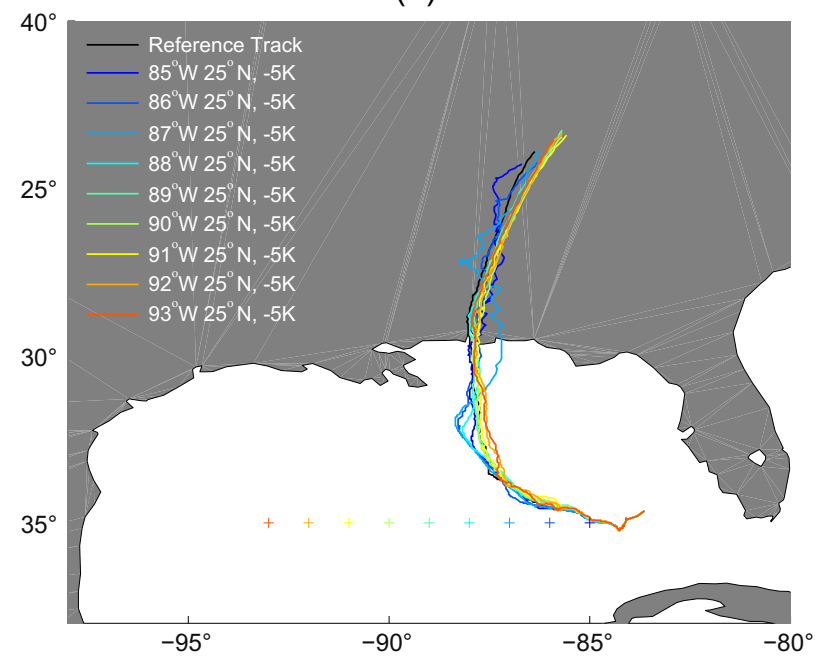

Figure 10: Hurricane trajectories of the reference simulation and the westwards localised SST experiments using an amplitude of (a) $+5 \mathrm{~K}$, and (b) $-5 \mathrm{~K}$.

point, cause an early increase of intensity. Additionally, the intensity remains higher than in the reference simulation during the entire simulation (Fig. 9a). The $+5 \mathrm{~K}$ anomaly at $85^{\circ} \mathrm{W} 25^{\circ} \mathrm{N}$ has the strongest impact on the intensity (up to $97 \mathrm{~m} \mathrm{~s}^{-1}$, as it is the closest to the initialisation point. For this simulation the maximum wind speed is reached after 26 hours, whereas the reference simulation shows its maximum after 48 hours. As already mentioned, negative SST anomalies cause a reduction of the intensity, when located nearby the initialisation point (Fig. 9b). The intensity is reduced after 6 hours for the experiment with a $-5 \mathrm{~K}$ anomaly at $85^{\circ} \mathrm{W} 25^{\circ} \mathrm{N}$ and the maximum is delayed by almost 12 hours compared to the reference experiment. Moreover, these experiments show that the strength is reduced in such a way that the vortex is classified as a tropical storm rather than a hurricane.

The response in the landfall already suggests a change in the trajectory of the vortex due to the SST anomalies. 

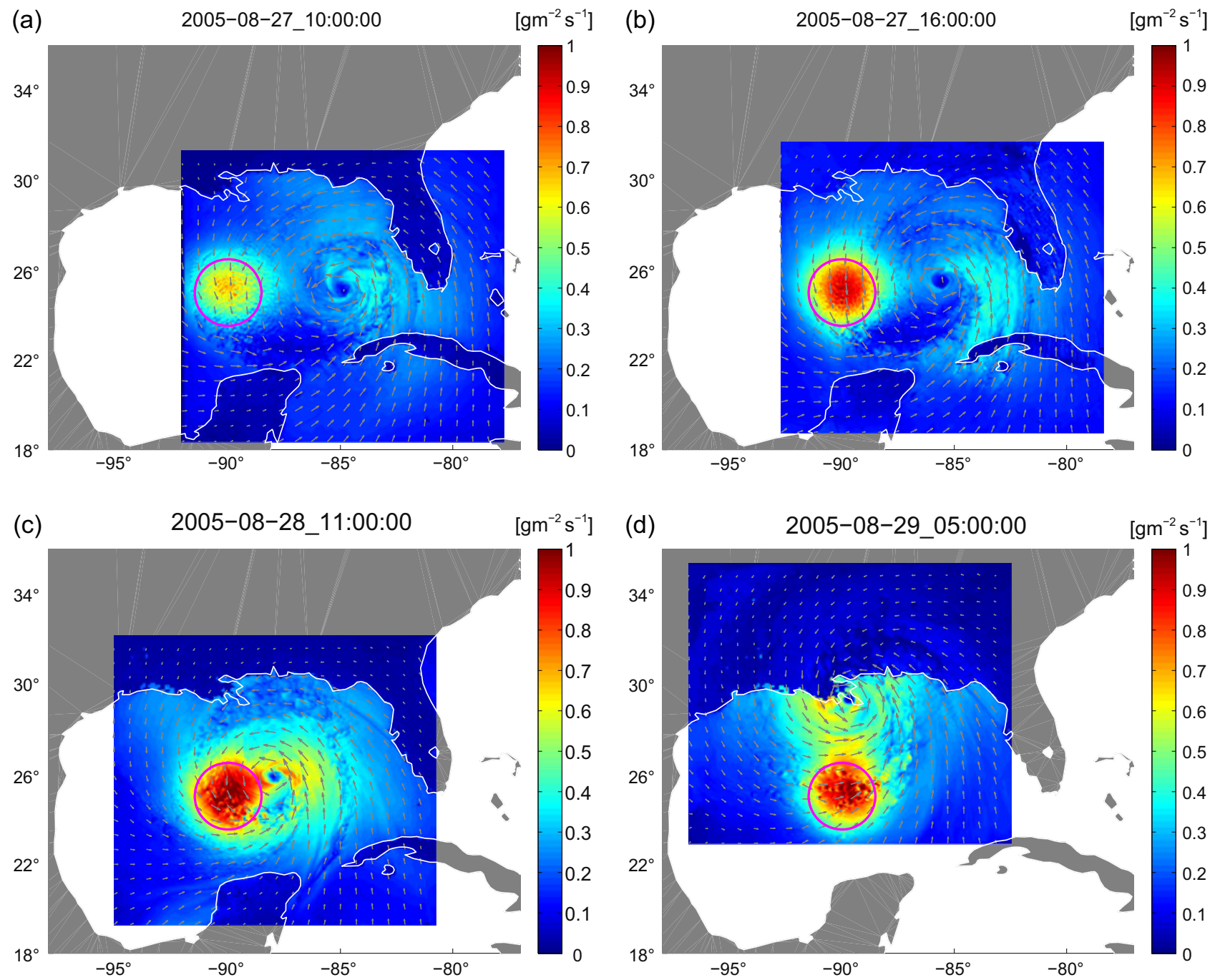

Figure 11: The upward moisture flux at the surface displayed for a SST anomaly experiment localised at $90^{\circ} \mathrm{W} 25^{\circ} \mathrm{N}$ with a SST amplitude of $+5 \mathrm{~K}$ at different time steps: (a) after 10 hours, (b) after 16 hours, (c) after 35 hours, and (d) after 53 hours. The location of the SST anomaly (Gaussian distributed) is marked by a circle and the arrows indicate the wind field at $10 \mathrm{~m}$. The panels represent the innermost domain (4 $\mathrm{km}$ resolution), which is centred around the vortex.

In Fig. 10a,b we show the trajectories of all experiments with a westwards located $\pm 5 \mathrm{~K} \mathrm{SST}$ anomaly. The strongest impact is found for positive SST anomalies, which are positioned westwards from the initialisation point (Fig. 10a). The maximum westward shift is achieved by the experiment with an anomaly located at $90^{\circ} \mathrm{W}$ $25^{\circ} \mathrm{N}$. Thus, the hurricane seems to be attracted by SST anomalies located in a certain distance range of $4^{\circ}$ to $8^{\circ}$ westwards to the initialisation point. The trajectories of the experiments with $-5 \mathrm{~K}$ show nearly no change, except for the ones where the anomaly is located nearby the initialisation point, which leads to a strong weakening so that the vortex partly loses its hurricane character, i.e., it is reduced to a tropical storm. In all other sensitivity experiments except for the westwards located SST anomaly experiments the trajectories are only weakly affected (therefore not discussed further; see Fig. 7c,d).
To understand the processes involved in the attraction of the trajectory, we exemplarily focus on the sensitivity experiment with the $+5 \mathrm{~K} \mathrm{SST}$ anomaly, which is located at $90^{\circ} \mathrm{W} 25^{\circ} \mathrm{N}$. The upward moisture flux at the surface is presented in the inner moving domain at four different times: $10 \mathrm{~h}, 16 \mathrm{~h}, 35 \mathrm{~h}$, and $53 \mathrm{~h}$ after initialisation (Fig. 11). As expected the SST anomaly causes a strong moisture flux, which starts interacting with the outer spiral rain bands at time 27.08.2005 at 10:00:00 UTC (Fig. 11a). The closer the hurricane approaches to the SST anomaly the stronger is the upward moisture flux at the surface (Fig. 11b,c). The reason is that higher wind speeds at the surface enhance evaporation. At time 29.08.2005 05:00:00 UTC the hurricane passes by the SST anomaly and the wind velocity above the SST anomaly is reduced and the upward moisture flux is decreased (Fig. 11d). Thus, the SST anomaly serves as an energy 
(a)

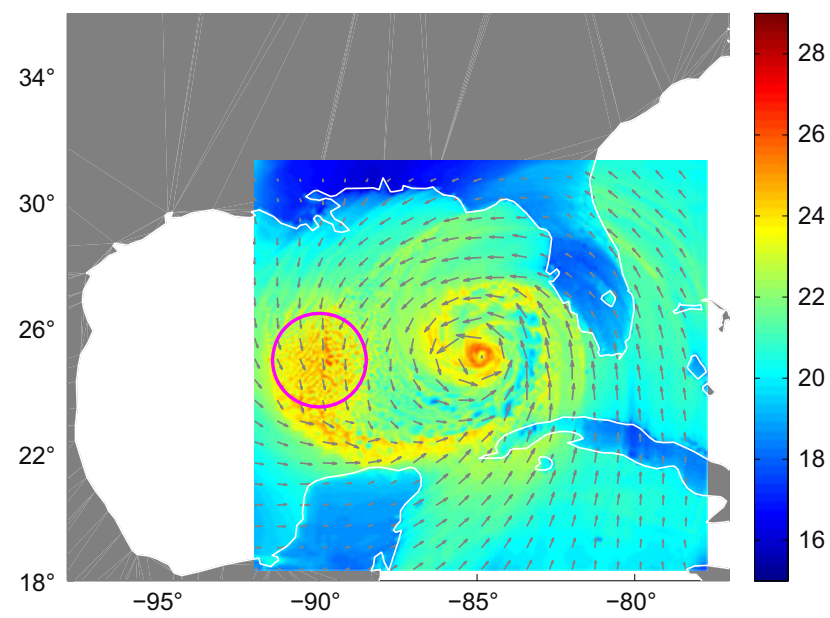

(c)

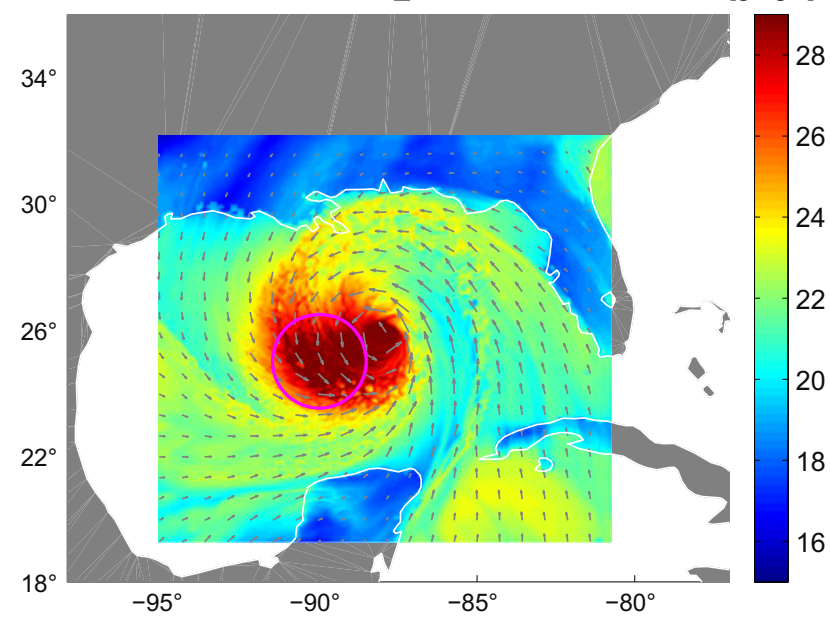

(b) 2005-08-27_16:00:00

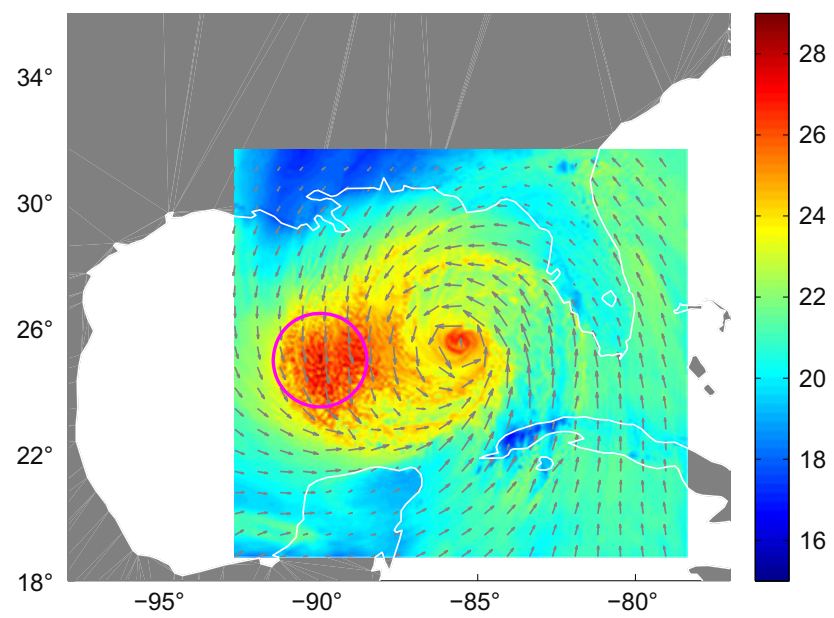

(d)

2005-08-29_05:00:00

$\left[\mathrm{kg}^{-1}\right]$

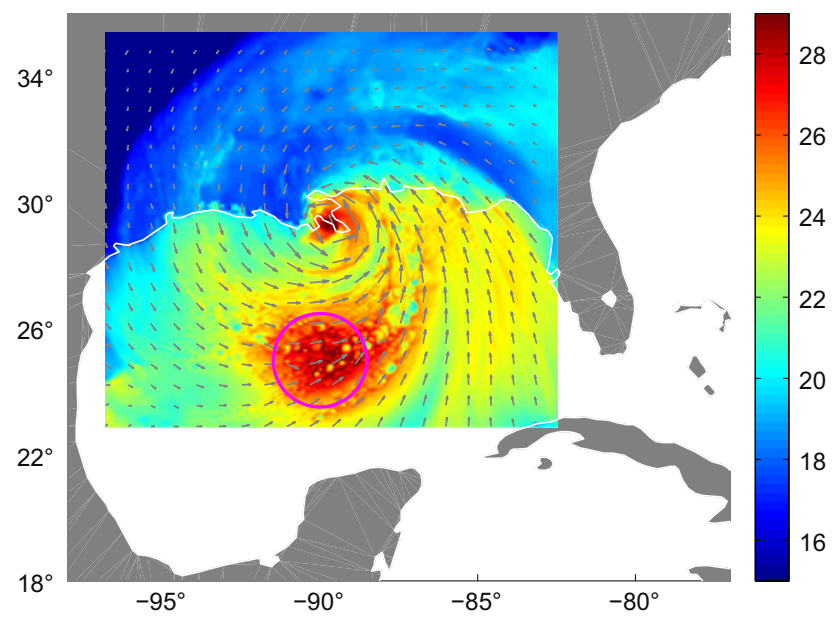

Figure 12: As Fig. 11, but for the water vapour mixing ratio at $2 \mathrm{~m}$.

source, where a positive temperature anomaly causes a higher upward moisture flux.

The upward moisture flux at the surface further increases with stronger horizontal wind enhancing the convection above the SST anomaly and thus leading to a stronger vertical wind field. Therefore, the vertical wind field above the SST anomaly is investigated and compared with the vertical wind field in the reference simulation at the corresponding distance and location to the TC centre (not shown). The finding is that the vertical wind is enhanced above the SST anomaly up to the tropopause compared to the reference simulation, even though the TC intensity in both simulations is similar. To confirm this finding the vertical wind field on the opposite side of the SST anomaly (on the line through SST anomaly and centre of the TC) is investigated in both simulations showing that vertical wind fields have the same strength. The increase in vertical wind above the SST anomaly induces a horizontal air flow to the direction of the SST anomaly. This leads to a deviation of the primary circulation (the horizontal axisymmetric circulation) of the hurricane towards the SST anomaly.

Fig. 12 presents the water vapour mixing ratio at $2 \mathrm{~m}$ for the same sensitivity experiment and time steps as Fig. 11. The 2-m mixing ratio illustrates how water vapour and thus latent heat is advected from the SST anomaly to the vortex (Fig. 12a). Besides the primary circulation also the secondary circulation (radial and vertical) of a hurricane is important. The additional water vapour over the SST anomaly leads to additional latent heat release. This heat release leads to an increase in updrafts and thus enhances the secondary circulation. The additional energy is used to increase the core speed of the TC.

To get a deeper understanding of the dynamics, the PVT is additionally investigated for the localised SST anomaly experiments. Therefore, we consider two experiments localised to the west $\left(\Delta 5^{\circ}\right.$ and $\Delta 1^{\circ}$, Fig. 2) with an amplitude of $+5 \mathrm{~K}$, respectively. The experiment with a SST anomaly $\Delta 5^{\circ}$ west has a significant influence on 
(a)

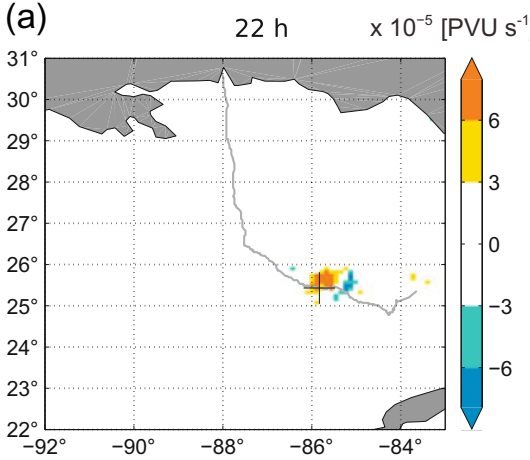

(d)

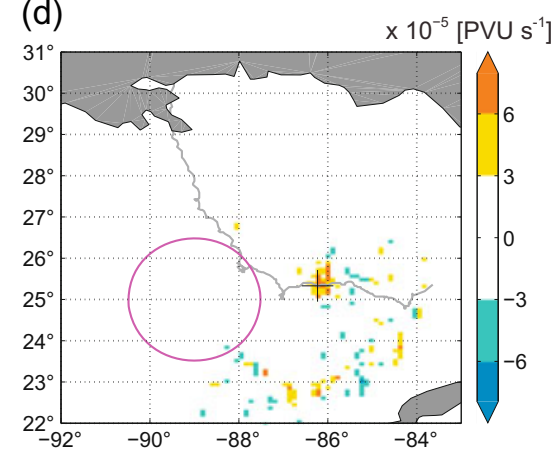

(g)

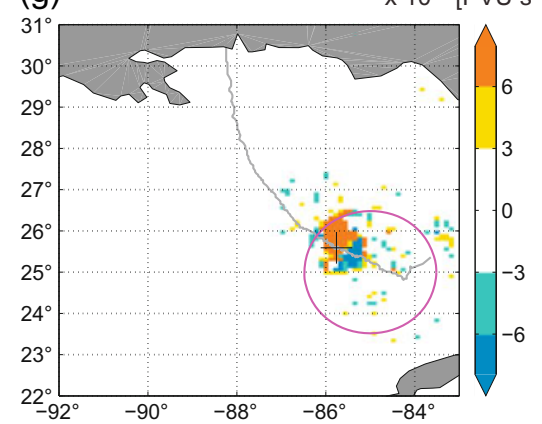

(b)

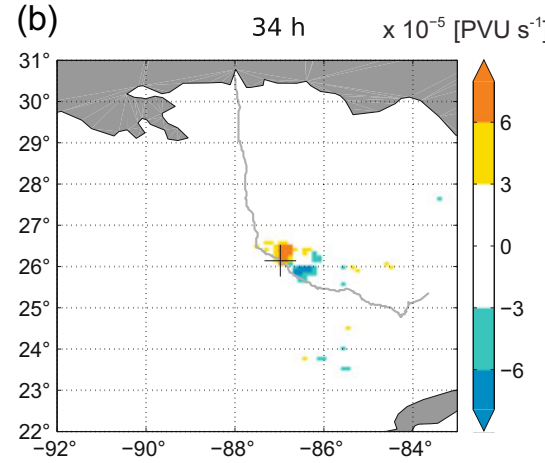

(e)

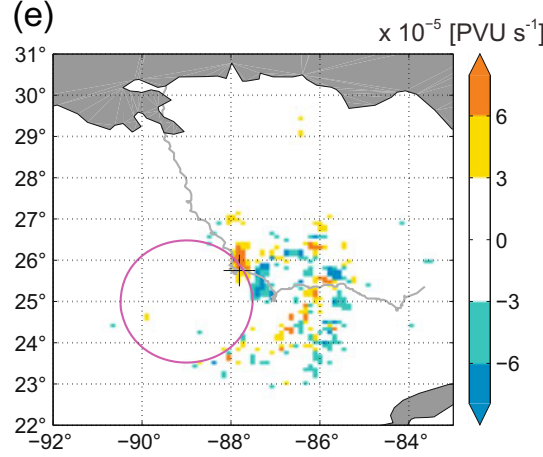

(h)

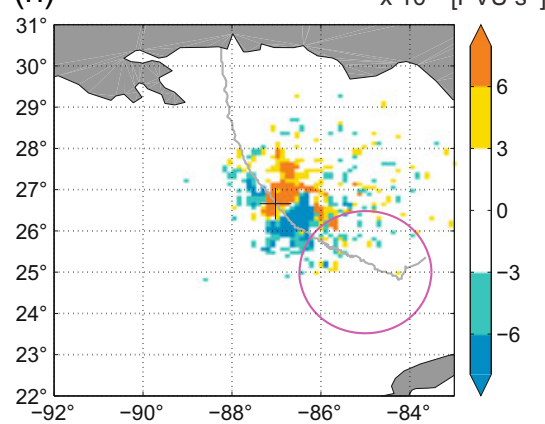

(c) $\quad 42 \mathrm{~h} \quad \times 10^{-5}\left[\mathrm{PVU} \mathrm{s}{ }^{-1}\right]$

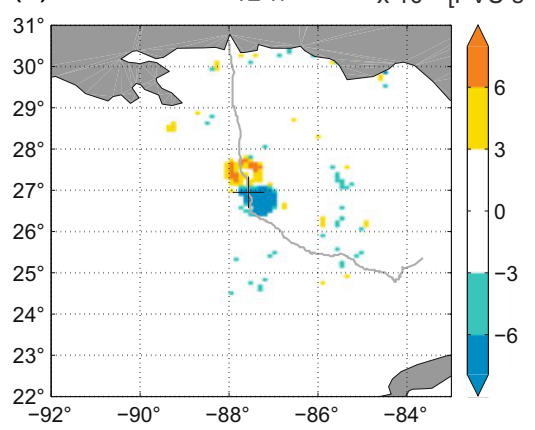

(f) $\times 10^{-5}\left[\mathrm{PVU} \mathrm{s}^{-1}\right]$

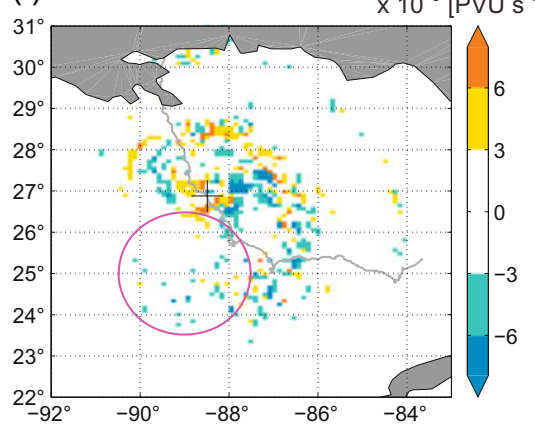

(i) $\quad \times 10^{-5}\left[\mathrm{PVU} \mathrm{s} \mathrm{s}^{-1}\right]$

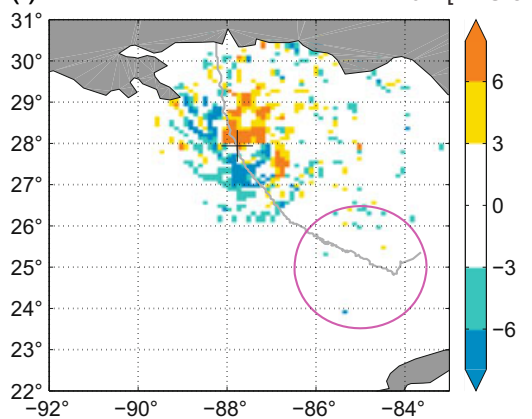

Figure 13: Diabatic heating at 22 h, 36 h, 42 h after initialisation at the lower layer (see Section 2) of (a-c) the reference simulation, the experiments using a localised SST anomaly (d-f) at $89^{\circ} \mathrm{W} 25^{\circ} \mathrm{N}$, and (g-h) at $85^{\circ} \mathrm{W} 25^{\circ} \mathrm{N}$ with an amplitude of $+5 \mathrm{~K}$, respectively.

TC trajectory (Fig. 7c, and 10a). In terms of magnitude and pattern, the horizontal advection term of PVT does not strongly differ from the reference simulation (therefore not shown). However, the pattern of diabatic heating term differs: A maximum of diabatic heating is still located in moving direction, but additionally the diabatic heating is found in the spiral rain bands (Fig. 13d-e). Thus, the vortex is drifted to the SST anomaly due to the asymmetry in the TC circulation induced by the diabatic heating term of the PVT. To assess the relation between PVT and intensity, we analyse the anomaly at $\Delta 1^{\circ}$ to the west (Fig. 2), which shows no effect on trajectory, but a strong impact on intensity (Fig. 7a, 8, and 9a). The SST anomaly below the cyclone centre effectively influences the diabatic heating term of PVT, which significantly increases compared to the reference simulation (Fig. $13 \mathrm{~g}$ ) and is able to enhance the Carnot cycle during the entire TC development (Fig. 13 g-i). This explains why the slope of the linear relation between TC intensity and SST changes (Fig. 8).

In summary localised positive and negative SST anomalies are able to influence the intensity. SST anomalies with a positive amplitude additionally have an effect on the trajectory.

\section{Discussion and conclusion}

By investigating the sensitivity experiments performed by basin-wide SST changes, we confirm the linear relationship between SST and TC intensity suggested by EMANUEL (1997), as a positive SST change strengthens the TC intensity by enhanced thermodynamic energy supply. Contrary to YUN et al. (2012) and CHAN et al. (2001), our simulations show that the rate of intensification 
does not decrease at SSTs above $305 \mathrm{~K}$. We detect a linear increase of storm intensity up to $307 \mathrm{~K}$. The mechanical energy is provided by Carnot processes and evaporation/latent heat fluxes. However, to understand the limitation of TC intensification further investigations are required. Additionally, the trajectory of the TC is shifted westwards with increasing SSTs. The reason for this change is that the background easterly wind is enhanced advecting the TC more effectively westwards. YUN et al. (2012) argued in their case study that the TC moves to the area of maximum PVT, which is consistent with our simulations, where the horizontal advection is the dominated process and the contribution of vertical advection is insignificant.

The influence of localised SST anomalies on TC trajectory and intensity is presented based on sets of sensitivity experiments for Hurricane Katrina $60 \mathrm{~h}$ before landfall. Our analysis confirms earlier modelling studies (MANDAL et al., 2007; YUN et al., 2012) and shows that SST anomalies have a major impact on intensity. Higher SSTs increase the intensity of the hurricane via diabatic processes. The impact of SST anomalies is clearly dependent on magnitude, distance, and direction of the anomaly with respect to the TC centre.

A TC is attracted by positive SST anomalies which is also found by MANDAL et al. (2007). In this study we show that positive SST anomalies enhance the vertical wind field caused by convection which shifts the horizontal wind field towards the SST anomaly. Hence, the primary circulation is affected by enhanced convection over the SST anomaly and the secondary circulation is enhanced by additional latent heat release causes the TC to move towards the SST anomaly. If positive anomalies are located in opposition to the motion direction, the contribution of the SST anomaly to the steering of the TC is small. Thus, the steering is dominated by the subtropical ridge and the beta effect. However, it is possible that positive anomalies behind the TC lead to a weakening of intensification. It has not been fully clarified yet, why this happens. Further, anomalies with a negative amplitude have no impact on the TC trajectory.

Comparing the time behavior of the intensity of the basin-wide sensitivity experiments with the ones of the localised SSTs changes we find some remarkable differences (Fig. 9). In contrast to the localised SST experiment the maximum intensity is reached approximately at the same time as in the reference experiment (Fig. 3). Moreover, the tropical cyclone reacts later (after 8 hours) to the underlying SST change, if this is uniformly distributed, whereas a localised SST change of similar amplitude $( \pm 2.5 \mathrm{~K})$ already shows an enhancement or reduction after 6 hours. The faster reaction of the vortex in terms of intensity in the localised SST experiments is related to the induced SST gradients. These gradients are absent when changing the SST field uniformly.

In summary, the study shows in a systematic way the impact of SST changes on intensity and trajectory in the case Hurricane Katrina. Still, this has to be confirmed in further case studies to obtain a better understanding of the impact of the physical environment on TCs.

\section{Acknowledgments}

We gratefully acknowledge discussions with Silke DIERER, Stefan MUTHERS, Andreas BORN, Niklaus MERZ, Flavio LEHNER, and Kay BIERI. Data storage is provided by the Swiss National Supercomputing Centre (CSCS). Christoph C. Raible is supported by the National Centre of Competence in Research (NCCR) Climate.

\section{References}

Alvera-AzCÁrAte, A., A. BARTH, R.H. WeISBERG, 2009: The surface circulation of the caribbean sea and the gulf of mexico as inferred from satellite altimetry. - J. Phys. Oceanogr. 39, 640-657.

Bengtsson, L., M. Botzet, M. Esch, 1996: Will greenhouse gas-induced warming over the next 50 years lead to higher frequency and greater intensity of hurricanes? Tellus A 48, 57-73.

CHAN, J.C.L., Y.H. DUAN, L.K. SHAY, 2001: Tropical cyclone intensity change from a simple ocean-atmosphere coupled model. - J. Atmos. Sci. 58, 154-172.

CHAN, J.C.L., F.M.F. KO, Y.M. LEI, 2002: Relationship between potential vorticity tendency and tropical cyclone motion. - J. Atmos. Sci. 59, 1317-1336.

CHANG, Y.L., L.Y. OEY, 2013: Loop current growth and eddy shedding using models and observations: Numerical process experiments and satellite altimetry data. - J. Phys. Ocean. 43, 669-689.

CiOnE, J.J., E.W. UhlHorn, 2003: Sea surface temperature variability in hurricanes: Implications with respect to intensity change. - Mon. Wea. Rev. 131, 1783-1796.

EMANUEL, K.A., 1997: Some aspects of hurricane inner-core dynamics and energetics. - J. Atmos. Sci. 54, 1014-1026.

EMANUEL, K., 2005: Increasing destructiveness of tropical cyclones over the past 30 years. - Nature 436, 686688.

Emanuel, K., C. Desautels, C. Holloway, R. Korty, 2004: Environmental control of tropical cyclone intensity. - J. Atmos. Sci. 61, 843-858.

EMANUEL, K., R. SUNDARARAJAN, J. Williams, 2008: Hurricanes and global warming - results from downscaling IPCC AR4 simulations. - Bull. Amer. Meteor. Soc. 89, 347-367.

Fredrick, S., C. DAVIS, D. GILl, S. LOW-NAM, 2009: Bogussing of tropical cyclones in WRF version 3.1. Technical report, National Center for Atmospheric Research (NCAR), Boulder, Colorado.

GARner, S.T., I.M. Held, T. KNUtSON, J. SiRutis, 2009: The roles of wind shear and thermal stratification in past and projected changes of Atlantic tropical cyclone activity. - J. Climate 22, 4723-4734.

HALl, W.D., R.M. RASMUSSEN, G. THOMPSON, 2005: The new Thompson microphysical scheme in WRF. 
- Technical report, National Center for Atmospheric Research (NCAR), Boulder, Colorado.

HONG, X., S.W. CHANG, S. RAMAN, L.K. SHAY, R. HOdUR, 2000: The interaction between hurricane opal (1995) and a warm core ring in the gulf of mexico. - Mon. Wea. Rev. 128, 1347-1365.

KAIN, J.S., 2004: The Kain-Fritsch convective parameterization: An update. - J. Appl. Meteor. 43, 170-181.

Kalnay, E., M. Kanamitsu, R. Kistler, W. Collins, D. DEAVEN, L. GANDin, M. IREDEll, S. SAHA, G. White, J. WoOllen, Y. ZHU, M. Chelliah, W. EBisuZaKi, W. Higgins, J. JANOWIAK, K.C. MO, C. ROPELEWSKI, J. WANG, A. LEETMAA, R. REYNOLDS, R. JENNE, D. JOSEPH, 1996: The NCEP/NCAR 40-year reanalysis project. - Bull. Amer. Meteor. Soc. 77, 437-471.

Kistler, R., E. KALNAY, W. COllins, S. SAHA, G. White, J. WoOllen, M. Chelliah, W. EbisuZaKi, M. KanamITSU, V. Kousky, van.den.H. DOOL, R. JENNE, M. FIORINO, 2001: The NCEP-NCAR 50-year reanalysis: Monthly means CD-ROM and documentation. - Bull. Amer. Meteor. Soc. 82, 247-267.

KLEPPEK, S., V. MuCCIONE, C.C. RAiBle, D.N. BRESCH, P. KOELLNER-HECK, T.F. STOCKER, 2008: Tropical cyclones in ERA-40: A detection and tracking method. - Geophys. Res. Lett. 35, L10705.

KNAPP, K.R., M.C. KRUK, D.H. LEVINSON, H.J. DiAMOND, C.J. NEUmanN, 2010: The international best track archive for climate stewardship (ibtracs) unifying tropical cyclone data. - Bull. Amer. Meteor. Soc. 91, 363-376.

Knutson, T.R., J.L. McBride, J. Chan, K. EMANuel, G. Holland, C. LANDSEA, I. Held, J.P. Kossin, A.K. SRIVASTAVA, M. SUgI, 2010: Tropical cyclones and climate change. - Nature Geoscience 3, 157-163.

LOW-NAM, S., C. DAVIS, 2001: The NCAR-AFWA tropical cyclone bogussing scheme. - Technical report, National Center for Atmospheric Research (NCAR), Boulder, Colorado.

LOW-NAM, S., C. DAVIS, 2009: Development of a tropical cyclone bogussing scheme for the MM5 system. Technical report, National Center for Atmospheric Research (NCAR), Boulder, Colorado.

MANDAL, M., U.C. MOHANTY, P. SINHA, M.M. Ali, 2007: Impact of sea surface temperature in modulating movement and intensity of tropical cyclones. - Natural Hazards 41, 413-427.
Raible, C.C., S. Kleppek, M. Wuest, D.N. Bresch, A. Kitoh, H. Murakami, T.F. Stocker, 2012: Atlantic hurricanes and associated insurance loss potentials in future climate scenarios: limitations of high-resolution AGCM simulations. - Tellus A 64, 15672.

SHAPIRO, L.J., J.L. FrANKLIN, 1995: Potential vorticity in hurricane Gloria. - Mon. Wea. Rev. 123, 1465-1475.

SHAPIRO, L.J., S.B. GOLDENBERG, 1998: Atlantic sea surface temperatures and tropical cyclone formation. - J. Climate 11, 578-590.

SKAMAROCK, W.C., J.B. KlemP, J. Dudhia, D.O. GILl, D.M. BARKER, M.G. DUdA, X.-Y. HUANG, W. WANG, J.G. POWERS, 2008: A description of the Advanced Research WRF version 3. - Technical report, NCAR Technical Note NCAR/TN-475+STR, National Center for Atmospheric Research (NCAR), Boulder, Colorado.

SRIVER, R., M. HUBER, 2006: Low frequency variability in globally integrated tropical cyclone power dissipation. Geophys. Res. Lett. 33, L11705.

SUGI, M., A. NODA, N. SATO, 2002: Influence of the global warming on tropical cyclone climatology: An experiment with the JMA global model. - J. Meteor. Soc. Japan 80, 249-272.

TRENBERTH, K.E., D.J. SHEA, 2006: Atlantic hurricanes and natural variability in 2005. - Geophys. Res. Lett. 33, L12704.

VECCHI, G.A., B.J. SODEN, 2007: Effect of remote sea surface temperature change on tropical cyclone potential intensity. - Nature 450, 1066-U9.

WANG, D.L., X.D. LianG, Y. ZhaO, B. WANG, 2008: A comparison of two tropical cyclone bogussing schemes. Wea. Forecast. 23, 194-204.

Webster, P.J., G.J. Holland, J.A. CuRry, H.R. Chang, 2005: Changes in tropical cyclone number, duration, and intensity in a warming environment. - Science 309, 1844 1846.

WU, L.G., B. WANG, 2000: A potential vorticity tendency diagnostic approach for tropical cyclone motion. - Mon. Wea. Rev. 128, 1899-1911.

YUN, K.S., J.C.L. CHAN, K.J. HA, 2012: Effects of SST magnitude and gradient on typhoon tracks around East Asia: A case study for Typhoon Maemi (2003). - Atmos. Res. 109, 36-51. 


\section{Appendix}

\section{Potential vorticity and potential vorticity}

\section{tendency}

The potential vorticity $P$ and its tendency $\frac{\partial P}{\partial t}$ are analysed similar to WU and WANG (2000), CHAN et al. (2002), and YUN et al. (2012):

$$
\begin{aligned}
P=-\frac{g}{p_{s}}\left[(\zeta+f) \frac{\partial \theta}{\partial \sigma}+\frac{\partial u}{\partial \sigma} \frac{\partial \theta}{\partial y}-\frac{\partial v}{\partial \sigma} \frac{\partial \theta}{\partial x}\right] & \frac{\partial P}{\partial t}=-u \frac{\partial P}{\partial x}-v \frac{\partial P}{\partial y}-\dot{\sigma} \frac{\partial P}{\partial \sigma} \\
+ & \frac{g}{p_{s}}\left[-(\zeta+f) \frac{\partial \dot{\theta}}{\partial \sigma}-\frac{\partial u}{\partial \sigma} \frac{\partial \dot{\theta}}{\partial y}+\frac{\partial v}{\partial \sigma} \frac{\partial \dot{\theta}}{\partial x}\right],
\end{aligned}
$$

where $g$ is the gravitational acceleration, $p_{s}$ the surface pressure, $\zeta$ the vorticity, $f$ the Coriolis parameter, $\theta$ the potential temperature, $\theta$ the rate of change of the potential temperature, $\sigma$ sigma coordinates, $\dot{\sigma}$ the vertical velocity in sigma coordinates, $u$ the zonal wind, $v$ the meridional wind, $x$ resolution in $\mathrm{x}$-direction, and $y$ resolution in $\mathrm{y}$ direction. The PVT (Eq. A.2) consists of the horizontal advection (first two terms), the vertical advection (third term), and the diabatic heating (the last term). 